\title{
Validation of the Thai version of the Duke Activity Status Index in patients with a previous myocardial infarction
}

\author{
Nisakorn Vibulchai ${ }^{\mathrm{a}}$, Sureeporn Thanasilpp, Sunida Preechawong ${ }^{\mathrm{a}}$, Marion E. Broome ${ }^{\mathrm{b}}$ \\ ${ }^{a}$ Faculty of Nursing, Chulalongkorn University, Bangkok 10330, Thailand, ${ }^{b}$ Indiana University School \\ of Nursing, Indianapolis, Indiana 46202, USA
}

\begin{abstract}
Background: The Duke Activity Status Index is a widely used instrument for measuring functional status in patients with cardiovascular disease. However, items and subscales on this instrument have not been validated for Thai patients with a previous myocardial infarction (MI).

Objective: To test the reliability and validity of the Thai version of the Duke Activity Status Index (DASI-T) in Thai patients with a previous MI using a cross-sectional study design.

Methods: The DASI-T was translated using forward and backward translation methods and administered to 100 MI patients from outpatient departments of two general hospitals in Thailand. Internal consistency was determined to test reliability. Two criterion measures (i.e. Canadian Cardiovascular Society (CCS) classification, SF-36 physical functioning subscale) were used to test the concurrent validity of the DASI-T. Age group and CCS classification were used to determine known-groups validity of the DASI-T.

Results: Cronbach's $\alpha$ for the DASI-T total score was 0.76 . No ceiling or floor effect was detected for the DASI-T total score. DASI-T total score was significantly correlated with the CCS classification $(r=-0.68, P<0.01)$ and SF-36 physical functioning subscale $(r=0.79, P<0.01)$. DASI-T total scores could differentiate MI patients based on age $(P=0.040)$ or CCS classification $(P=0.000)$.

Conclusion: The DASI-T is a potentially reliable and valid instrument with which to assess functional status in MI patients and is also useful to evaluate a treatment effect and be a guideline for clinical purposes (i.e. exercise prescription, risk stratification).
\end{abstract}

Keywords: Duke Activity Status Index, myocardial infarction, reliability, validity

Myocardial infarction (MI), an acute manifestation of coronary artery disease, has become a serious health problem worldwide. Although there are fewer patients dying in the acute stage of the illness because of recent advances in medical treatment of myocardial infarction, a large number of MI patients fail to resume normal functioning. Rather, they experience a decline in functional status [1-3].

Goals of care for MI patients include optimizing cardiovascular risk reduction, fostering healthy behaviors and compliance to these behaviors, reducing disability, and promoting an active lifestyle for patients [4]. It is important to use reliable and valid measurements to assessing progress towards these goals in practice. One such method of measurement, the Duke Activity Status Index (DASI), was developed to assess the functional status of patients

Correspondence to: Sureeporn Thanasilp, Faculty of Nursing, Chulalongkorn University, Bangkok 10330, Thailand. E-mail: s_thanasilp@hotmail.com with cardiovascular disease, and to measure a patient's maximum physical potential for performing common activities of daily living [5]. Currently, the DASI is a widely used instrument for measuring functional status [6-8]. However, the items and subscales on the DASI have not been validated for the psychometric testing of Thai patients with cardiovascular disease. To understand the impact of disease conditions on functional status across cultures, there is a need for this instrument to be translated and its psychometric properties assessed in a Thai cultural context.

The purpose of this study was to test the reliability and validity of the Thai version of the Duke Activity Status Index (DASI-T) in assessing the functional status of Thai patients with a previous MI.

\section{Materials and methods \\ Design, sample, and setting}

A cross-sectional design was used to evaluate the validity and reliability of the DASI-T in a sample of patients who had experienced an MI. After 
approval by the Research Ethics Committees of Mahasarakham and Kalasin Hospitals, Thailand, data were collected during June and July 2013. A convenient sample of 100 patients with a previous MI was recruited from outpatient departments after their written informed consent to participate in the study. The patient inclusion criteria were as follows: (1) patients diagnosed with either an ST-elevation MI (STEMI) or a non ST-elevation MI (NSTEMI); (2) stable disease, defined as no hospitalization for MI in the previous four weeks; and (3) willingness to participate in the investigation. The exclusion criteria included: (1) having a complication that could influence daily activity performance such as congestive heart failure, uncontrolled hypertension (systolic blood pressure $>180 \mathrm{mmHg}$ and diastolic blood pressure $>110 \mathrm{mmHg}$, or both), uncontrolled hypotension (diastolic BP $<65 \mathrm{mmHg}$ ), chronic obstructive pulmonary disease or history of asthma; (2) health problems including physical problems (e.g. musculoskeletal, neurological, rheumatic diseases with functional impairment), or mental problems (e.g. severe anxiety, depression).

\section{Measurement}

Three instruments were used in this study: the Thai version of the Duke Activity Status Index (DASIT); the Canadian Cardiovascular Society (CCS) classification [9]; and the SF-36 physical functioning subscale $[10,11]$. The CCS classification and the SF-36 physical functioning subscale were used as standard measurements to determine concurrent validity of the DASI-T. Age groups ( $<60$ years, $\geq 60$ years) and functional class by the CCS classification were also used as a criterion variable to determine known-group validity of the DASI-T based on the extensive review of age and functional class related to functional status. Older MI patients were more likely to have impaired physical ability performance in their daily lives [12, 13]; increasing CCS class was inversely related to an MI patient's maximum physical potential to perform common activities of daily living [14] and associated with poorer treadmill performance [15].

\section{The Thai version of the Duke Activity Status Index (DASI-T)}

The DASI-T is a self-administered 12-item measure derived from the Duke Activity Status Index (DASI) developed by Hlatky and colleagues [5]. The
DASI-T is congruent with the original DASI. There are four major activity domains: personal care, ambulation, household tasks, sexual function, and recreation. Each patient participant was asked whether they could perform each activity. The response format for this was "Yes"/"No". If the patient stated "Yes," that item was then assigned a weighted score based on the known metabolic cost of each activity. The weighted range score for each activity was 1.75-8.0. If the patient stated that they could not perform an activity ("No"), then the weighted score was zero. The potential range of the sum score is 0-58.2: 0 = worst, 58.2 = best; higher scores indicate better functional capacity [5]. The sum score can be applied to an estimation of maximal oxygen consumption $\left(\mathrm{VO}_{2}\right)$, the following multiple linear regression equation was used [5]: $\mathrm{VO}_{2}=0.43 \times$ DASI + 9.6, metabolic equivalents (METs) were calculated by dividing by 3.5 ; the resting or basal oxygen consumption $\left(\mathrm{VO}_{2}\right)$ of a $70 \mathrm{~kg}, 40$-year-old man in a resting state is $3.5 \mathrm{~mL}$ per $\mathrm{kg}$ per min, or $1 \mathrm{MET}$ [16]. A series of cut-off points in the DASI-T were used to determine the level of functional capacity based on metabolic equivalents (METs) [16]: less than 4 METs (poor), 4 to 7 METs (moderate), and $\geq 7$ METs (good). For this study, the original DASI English version was translated into Thai using a forward-backward method by a panel of experts and by interviewing a small sample of MI patients. The translation process was implemented as recommended by the International Society for Pharmacoeconomics and Outcomes Research (ISPOR) Task Force on the Translation and Cultural Adaptation Process for Patient-Reported Outcome (PRO) Measures [17]. The forward translation process began by the translation of the original English version of the DASI into Thai language by two bilingual native Thai nursing faculty members who were experienced in instrument development, and familiar with the target population. The DASI-T was then reviewed by the original translators, two physicians who specialize in cardiac rehabilitation, and a researcher. These experts were asked to examine each item for comprehension or ambiguity of wording. Some wording on the items was replaced with other wording that was conceptually equivalent and wellknown to Thai people, for example, 'walk a block or 2 on level ground' was replaced with 'walk around 3-4 electricity posts (80-160 meters)'. The instrument was then back translated to English by one bilingual person who was unfamiliar with the original English version, 
but had adequate knowledge of, and experience with, translation from Thai to English. The previous expert panel reviewed the back-translated questionnaire in relation to the original questionnaire to ensure linguistic and conceptual equivalence. Finally, the researcher pretested the translated version by administering it to five MI patients to identify any semantic or comprehension difficulties.

\section{The Canadian Cardiovascular Society Classification}

The Canadian Cardiovascular Society (CCS) classification is commonly used by clinicians for functional classification based on symptoms of angina and activity limitations [9]. The CCS classification is comprised of four descriptive classes (class I-IV). The classes range from no limitation of ordinary activity (Class I) to being unable to carry on any physical activity without discomfort (Class IV) [9]. Patients are placed in one of four descriptive classes. The psychometric properties of the CCS classification were tested in cardiovascular patients [15], the concurrent validity (percentage of functional class estimates that agreed with the treadmill performance class) was $59 \%$ and the kappa statistic value was 0.47 . The CCS classification had the strongest Spearman rank correlation $\left(r_{s}=-0.64\right)$ with the duration of the treadmill exercise in seconds. Reliability testing of the CCS classification has a documented reproducibility (interobserver agreement) of $73 \%$ and kappa statistic value of 0.60 [15].

\section{The SF-36 physical functioning subscale}

The SF-36 physical functioning subscale is a 10 item questionnaire from the version 2.0 of the SF-36 (SF-36v2), which measures a person's perceived limitations related to physical functioning because of a health problem [10,11]. Patients were asked if their health limits physical activity, basic mobility, and basic activities of daily living. Patients were asked to rate their limitations at the present time. Each item was rated on a 3-point Likert scale (yes, limited a lot; yes, limited a little; and no, not limited at all). For scoring and interpretation, all questions were summed to produce raw scores and then transformed to a 0-100 scale; higher scores representing better health status $[10,11]$.

The SF-36 physical functioning subscale is available in more than 170 translations [10]. In Thailand, the psychometric properties of the retranslated SF-36 (Thai version) subscale have been tested in 448 people by Leurmarnkul and Meetam with an acceptable construct validity and a high $\alpha$ coefficient (0.78) [18].

\section{Data collection}

Explanations of the purpose of the study, procedural details, the content of the questionnaire, the participant's rights, and the potential benefits and risks of the study were given to patients. After written informed consent was obtained from a patient, clinical data was collected from the medical records of the patient participant. Then, the researcher asked eligible patient participants to complete a packet of questionnaires. The patient participants took about 15-20 minutes to complete the questionnaires. The final sample size was 100 patient participants with a previous MI.

\section{Statistical analysis}

Statistical analyses were completed using the Statistical Package for the Social Sciences (SPSS), version 20. Descriptive statistics are presented as percentages, mean plus or minus $( \pm)$ standard deviation (SD), and as interquartile ranges. Internal consistency was assessed using Cronbach's $\alpha$. Ceiling and floor effects were determined by calculating the percentage of participants with the highest and lowest possible scores.

To assess known-group validity, an independent $t$ test was used to compare DASI-T total scores by age group (age $<60$ years, age $\geq 60$ years). Analysis of variance (ANOVA) was used to compare DASI$\mathrm{T}$ total scores by CCS classification. To assess concurrent validity, a Spearman correlation coefficient $\left(r_{s}\right)$ was used to assess the association among the DASI-T total scores and categories of patients according to the CCS classification; Pearson's correlation coefficient $(r)$ was used to assess the association between the DASI-T total score and the SF-36 total score physical functioning subscale. All statistical tests were performed at the two-tailed 5\% level of significance.

\section{Results \\ Description of the sample}

Demographic characteristics of the study participants are summarized as follows. The age of participants ranged from 38 to 85 years with a mean age of 63.99 years (SD 11.03). Sixty-four MI patients 
(64\%) were elderly ( $\geq 60$ years), $36 \%$ were adult (age $<60$ years). The majority of the participants were male (57\%), diagnosed with NSTEMI (76\%), receiving only medical therapy (94\%), and having multiple comorbidities: hypertension (50\%), diabetes mellitus (35\%), and dyslipidemia (33\%). The participants had an average of a body mass index more than $25 \mathrm{~kg} / \mathrm{m}^{2}$ (29\%), some are current smokers (14\%) or exsmokers (39\%), and about 39\% experienced chest pain sometimes with an average of 1-2 times/month.

\section{Description of scores on measures}

The potential range on the DASI-T total score was 0 -58.2. In this sample the range of total scores was 4.5-53.2, with a mean score of 27.51 (SD 12.94), indicating that this sample had a moderate level of physical functioning. For the SF-36 physical functioning subscale, the range of total score was 40-96.67, with a mean score of 68.10 (SD 15.84). This mean score indicates a moderate level of physical functioning.

Descriptive statistics for the DASI-T by age group and CCS classification are presented in Table 1. Mean DASI-T total score was higher for MI patients with age $<60$ years than for MI patients with age $\geq 60$ years. For the CCS classification, class I MI patients had the highest mean DASI-T score (35.43 \pm 10.80$)$ and class III MI patients had the lowest (11.17 \pm 5.85$)$. The lower DASI-T scores were found in patients with a higher class of MI.

\section{Reliability}

Cronbach's $\alpha$ coefficient for the DASI-T was 0.76 , indicating a high reliability. For the SF-36 physical functioning subscale, Cronbach's $\alpha$ coefficient was also high (0.89). The percentage of MI patients obtaining the high scores (ceiling effect) or low scores (floor effect) for the DASI-T total scores and the SF-36 physical functioning subscale was lower than $15 \%$, which suggests that there were no ceiling or floor effects to the reliability of those instruments [19].

\section{Validity}

Known-group validity

We found that there were significant differences in DASI-T total scores across age groups and CCS classification as represented in Table 1. These differences mean the DASI-T is able to distinguish between patients in different age groups and CCS classification. The DASI total score for MI patients with age $<60$ years was significantly higher than that for those MI patients with age $\geq 60$ years. Also, multiple pairwise comparisons between class I and class II, class I and III, and class II and class III revealed the DASI-T total score were significantly different.

\section{Concurrent validity}

The associations between the DASI-T and criterion measures were statistically significant (Table 2). There was a moderate negative correlation between the DASI-T total score and CCS classification. This correlation suggests that MI patients with a lower DASI-T score were more likely to be in a higher CCS classification. The DASI-T total score was highly positively correlated with the SF-36 physical functioning subscale. That is, MI patients with higher DASI-T scores also reported higher scores on the SF-36 physical functioning subscale.

Table 1. Scores of DASI-T by age group and CCS classification

\begin{tabular}{lll}
\hline Group & DASI-T $(\mathbf{m} \pm \mathbf{S D})$ & $\boldsymbol{P}$ \\
\hline${ }^{\text {tAge }}$ & & 0.040 \\
$\quad<60$ years $(\mathrm{n}=36)$ & $31.05( \pm 11.28)$ & \\
$\geq 60$ years $(\mathrm{n}=64)$ & $25.52( \pm 13.46)$ & 0.000 \\
${ }^{\mathrm{a} C \text { CS classification }}$ & & \\
$\quad$ Class I $(\mathrm{n}=44)$ & $35.43( \pm 10.80)$ & \\
Class II $(\mathrm{n}=33)$ & $28.34( \pm 7.65)$ & 0.004 \\
Class III $(\mathrm{n}=23)$ & $11.17(5.85)$ & 0.000 \\
Class I vs Class II & & 0.000 \\
Class I vs Class III & & \\
Class II vs Class III & &
\end{tabular}

m, Mean; SD, standard deviation; ${ }^{\mathrm{t}}=t$ test; ${ }^{\mathrm{a}}=$ Analysis of variance (ANOVA); DASI-T, Duke Activity Status Index-Thai version, CCS = Canadian Cardiovascular Society classification 
Table 2. Coefficients of correlation between the DASI-T and validating measures

\begin{tabular}{lcc}
\hline \multicolumn{1}{c}{ CCS classification } & ${ }^{+}$SF-36 physical functioning subscale \\
\hline DASI-T total score & $-0.68^{*}$ & $0.79 *$ \\
\hline${ }^{\#}$ Spearman correlation coefficient, ${ }^{+}$Pearson correlation coefficient, ${ }^{*} P<0.01$; DASI-T, Duke Activity \\
Status Index-Thai Version, CCS, Canadian Cardiovascular Society
\end{tabular}

\section{Discussion}

This study was conducted to validate whether the DASI-T could be used to measure functional status in Thai patients with MI. We used a rigorous validation process that began by clarifying the concept of functional status, reviewing established instruments to measure this concept in patients with MI, and selecting instruments related to defining functional status. The DASI was the instrument selected because it was designed to measure functional status in heart patients involving perception of their functional capacity in terms of activities that individuals perform in their daily lives [5]. A process of cross-cultural investigation was then used based on the ISPOR task force on translation guidelines [17], which use a team approach with independent bilingual translators to highlight problems in the translated measures including vocabulary, experiential, and conceptual equivalence. The process of translation in this study yielded content equivalence between the DASI-T and the original version.

\section{Reliability}

Internal consistency was based on the average correlation between items within the test [20]. Nunnally suggests that if the internal consistency of a new instrument is lower than 0.7 , the instrument should be reconsidered [20]. In this study, the internal consistency of Cronbach's $\alpha$ coefficient of the DASIT was acceptable (0.76). A "high" value of $\alpha$ is used as evidence that the items measure an underlying (or latent) construct [21]. The result of the internal consistency in this study is similar to that of a previous study [22], which revealed a high value for Cronbach's $\alpha$ coefficients (0.81-0.89). A ceiling or floor effect for the DASI-T total score was not detected in this study, which means no effect of the highest or lowest score rendered the questionnaire inadequate for assessing functional status. This evidence indicated the DASI-T is a reliable tool to measure functional status in MI patients.

\section{Validity}

Evidence of adequate concurrent validity was found in the association between the DASI-T and the criterion measures. The level of a significant correlation between the DASI-T total score and the CCS classification was moderate $\left(r_{s}=-0.68, P<0.01\right)$. This result was congruent with previous findings that showed a significant correlation between these two instruments [14, 22]. Similarly, a significant high correlation was found between the DASI-T total score and the total score of the SF-36 physical functioning subscale $(r=0.79, P<0.01)$, supporting a correspondence to an individual's ability to perform common activities of daily living. Known-group validity was also found in the DASI-T. Known-group validity is a subtype of construct validity that is demonstrated when individuals from populations that are 'known' to be 'high' (or 'low') on the construct being assessed score higher (or lower) on the purported measure of that construct than individuals 'known' to be 'low' (or 'high') on that construct [21]. In DASI-T known-group validity testing, DASI-T total scores by age groups and CCS classification were found to be significantly different; the differences suggest that the DASI-T is a valid measure for determining different scores in different clinical characteristics related to the functional status of samples. This finding is similar to that in a recent study by Mantziari and colleagues, who found significantly different DASI scores across age groups [8]. Together, this evidence supports the validity of the DASI-T as a measure of functional status for patients with MI.

\section{Limitations and recommendations}

One limitation was the participants in the study represented a select population of patients with myocardial infarction from only two settings. Future studies could use the DASI-T to measure functional status across all cardiovascular patients and to select patients by random sampling to generalize the results to the larger population. A second limitation is that 
the different numbers of CCS class I, II and class III patients may increase the variability of the estimates. Future studies may consider the proportion of patients in the CCS classification. Another limitation is that the CCS classification was evaluated by nurses in two different settings, and it is possible that the definitions were interpreted differently by these nurses. Future studies should measure inter-rater and intrarater reliability for assessing the CCS classification before the DASI-T is validated. Finally, the data on the DASI-T, CCS classification, and SF-36 physical functioning subscale were obtained at only one time point. Future studies should test the responsiveness of the DASI-T to clinical changes in MI patients over time.

\section{Conclusions}

The DASI-T appears to be a potentially useful, reliable, and valid instrument to assess functional status in terms of functional capacity in patients with MI. The DASI-T values can be used to evaluate treatment for patients with MI and act as a clinical guide (i.e. exercise prescription, risk stratification).

\section{Acknowledgments}

This study was funded, in part, by the $90^{\text {th }}$ Anniversary of Chulalongkorn University Fund (Ratchadaphiseksomphot Endowment Fund). The authors thank all the patients who participated in this study. A thank you is also extended to Professor Mark A. Hlatky, MD, for his generous permission for translation of the "Duke Activity Status Index," and to Jingwei Wu, Biostatistician, School of Medicine, Indiana University, for his statistical assistance in the initial preparation of the manuscript. None of the authors have any conflict of interest to report.

\section{References}

1. LaPier TK. Functional status during immediate recovery after hospitalization for coronary heart disease. J Cardiopulm Rehab. 2003; 23:203-7.

2. Jarrell LA, Hains, SJ, Kisilevsky BS, Brown CA. Gender differences in functional capacity following myocardial infarction: an exploratory study. Can J Cardiovasc Nurs. 2005; 15:28-33.

3. Dodson JA, Arnold SV, Reid KJ, Gill TM, Rich MW, Masoudi FA, et al. Physical function and independence 1 year after myocardial infarction: observations from the Translational Research Investigating Underlying disparities in recovery from acute Myocardial infarction: Patients' Health status registry. Am Heart J. 2012; 163:790-6.

4. Balady GJ, Ades PA, Comoss P, Limacher M, Pina IL, Southard D, et al. Core components of cardiac rehabilitation/secondary prevention programs. Circulation. 2000; 102:1069-73.

5. Hlatky MA, Boineau RE, Higginbotham MB, Lee KL, Mark DB, Califf RM, et al. A brief self-administered questionnaire to determine functional capability (The Duke Activity Status Index). Am J Cardiol. 1989; 64:651-4.

6. Phillips L, Wang JW, Pfeffer B, Gianos E, Fisher D, Shaw LJ, et al. Clinical role of the Duke Activity Status Index in the selection of the optimal type of stress myocardial perfusion imaging study in patients with known or suspected ischemic heart disease. J Nucl Cardiol. 2011; 18:1015-20.

7. Brual J, Gravely S, Suskin N, Stewart DE, Grace SL. The role of clinical and geographic factors in the use of hospital versus home-based cardiac rehabilitation. Int J Rehabil Res. 2012; 35:220-6.

8. Mantziari L, Kamperidis V, Ventoulis I, Damvopoulou E, Giannakoulas G, Efthimiadis G, et al. Increased right atrial volume index predicts low Duke Activity Status Index in patients with chronic heart failure. Hellenic J Cardiol. 2013; 54: 32-38.

9. Campeau L. Grading of angina pectoris. Circulation. 1976; 54:522-3.

10. Ware JE. SF-36 Health Survey update. [online]. [cited 2013 January 19]. Available from: http://www.sf-36. org/tools/sf36.shtml

11. Ware JE, Sherbourne CD. The MOS 36-Item ShortForm Health Survey 1: conceptual framework and item selection. Med Care. 1992; 30:473-83.

12. Sindhu S, Sriprasong S. A study of physical health conditions and level of activity during the recovering phase after discharge of acute myocardial infarction patients. The Thai Journal of Nursing Council. 2001; 16:52-68. [in Thai].

13. Hofer S, Benzer W, Alber H, Ruttmann E, Kopp M, Schussler G, et al. Determinates of health-related quality of life in coronary artery disease patients: a prospective study generating a structural equation model. Psychosomatics. 2005; 46:212-23.

14. Kaul P, Naylor CD, Armstrong PW, Mark DB, Theroux P, Dagenais GR. Assessment of activity status and survival according to the Canadian Cardiovascular Society angina classification. Can J Cardiol. 2009; 25: e225-31.

15. Goldman L, Hashimoto B, Cook EF, Loscalzo A. 
Comparative reproducibility and validity of systems for assessing cardiovascular functional class: advantages of a new specific activity scale. Circulation. 1981; 64:1227-34.

16. Fleisher LA, Beckman JA, Brown KA, Calkins H, Chaikof EL, Fleischmann KE, et al. ACC/AHA 2007 guidelines on perioperative cardiovascular evaluation and care for noncardiac surgery: a report of the American College of Cardiology/American Heart Association Task Force on Practice Guidelines (Writing Committee to Revise the 2002 Guidelines on Perioperative Cardiovascular Evaluation for Noncardiac Surgery). Circulation. 2007; 116:e418-500.

17. Wild D, Grove A, Martin M, Eremenco S, McElroy S, Verjee-Lorenz A, et al. Principles of good practice for the translation and cultural adaptation process for patient-reported outcomes (PRO) measures: report of the ISPOR Task Force for Translation and Cultural Adaptation. Value Health. 2005; 8:94-104.
18. Leurmarnkul W, Meetam P. Properties testing of the retranslated SF-36 (Thai version). Thai J Pharm Sci. 2005; 29:69-88.

19. McHorney CA, Tarlov AR. Individual-patient monitoring in clinical practice: are available health status surveys adequate? Qual Life Res. 1995; 4: 293-307.

20. Nunnally JC, Bernstein I. Psychometric Theory. 3rd ed. New York: McGraw-Hill; 1994.

21. DeVellis RF. Scale development: theory and applications. 2nd ed. California: Sage Publications; 2003.

22. Alonso J, Permanyer-Miralda G, Cascant P, Brotons C, Prieto L, Soler-Soler J. Measuring functional status of chronic coronary patients. Reliability, validity and responsiveness to clinical change of the reduced version of the Duke Activity Status Index (DASI). Eur Heart J. 1 